\title{
THE MAGNETIC FIELD OF THE INTERMEDIATE POLAR RE 0751+14
}

\author{
H. VÄTH \\ Institut für Astronomie und Astrophysik der Universität, 24098 \\ Kiel, Germany (supas097@astrophysik.uni-kiel.d400.de)
}

Piirola, Hakala \& Coyne (1993) modeled the optical/IR light curve of RE 0751+14 assuming a uniform shock structure and neglecting the hard $\mathrm{X}$-ray emission. In this paper, we model the light curves at optical/IR and hard X-ray wavelengths and include the effects of the shock structure.

We base our model on accretion onto a white dwarf with a displaced magnetic dipole for a range of likely white dwarf masses. We find that the observed intensity variations of X-rays and in the $I$ band over one spin period largely determine the position of the emission regions. Furthermore, the observed maximum X-ray flux constrains the specific accretion rate. We deduce that the magnetic field at the pole is likely to be in the range $9 \ldots 21 \mathrm{MG}$, which is consistent with the estimates of Piirola et al. (1993). It had been proposed previously that there must exist asynchronous rotators with sufficiently strong magnetic fields such that the binaries will evolve into AM Her binaries (Chanmugam \& Ray 1984; King, Frank \& Ritter 1985). With this deduced high magnetic field RE $0751+14$ is the most likely example of such a system known to date.

For more details see Väth, Chanmugam \& Frank (1996).

Acknowledgements. The author thanks Ganesh Chanmugam and Juhan Frank for their contribution to this work. This work was supported by NASA grant NAGW 2447 to LSU. The calculations were performed on the MasPar computer of the Department of Physics and Astronomy, Baton Rouge, USA and on the MasPar computer of the University of Karlsruhe, Germany.

\section{References}

Chanmugam, G., Ray, A., 1984, Ap. J., 285, 252

King, A.R., Frank, J., Ritter, H., 1985, MNRAS, 213, 181

Piirola, V., Hakala, P., Coyne, G.V., 1993, Ap. J., 410, L107

Väth, H., Chanmugam, G., Frank, J., 1996, Ap. J., in press

183

A. Evans and J. H. Wood (eds.), Cataclysmic Variables and Related Objects, 183.

(c) 1996 Kluwer Academic Publishers. Printed in the Netherlands. 